\title{
La naturaleza ontogenética, psicológica y epistemológica de los errores y obstáculos en la construcción de hipótesis científicas: un estudio de caso \\ The ontogenetic, psychological, and epistemological nature of errors and obstacles in the construction of scientific hypotheses: a case study
}

\author{
Dení Stincer-Gómez ${ }^{1,2}$ (1) http://orcid.org/0000-0003-3884-6805 \\ Ana Claudia Couló ${ }^{3}$ (i) https://orcid.org/0000-0002-7351-5596 \\ Zuraya Monroy Nasr ${ }^{2}$ (D) https://orcid.org/0000-0002-3837-5735 \\ ${ }^{1}$ Universidad Autónoma del Estado de Morelos, ${ }^{凶}$ dstincerg@gmail.com \\ ${ }^{2}$ Universidad Nacional Autónoma de México \\ ${ }^{3}$ Universidad de Buenos Aires \\ (C) Universidad De La Salle Bajío (México)
}

Recibido en: 18 - 09-2020 / Aceptado en: 16 - $02-2021$

\section{Resumen}

Un análisis del discurso argumentativo de estudiantes de posgrado de seis generaciones (seis años escolares) de la Facultad de Psicología de la Universidad Nacional Autónoma de México, nos muestra un conjunto de obstáculos y errores sistemáticos en el proceso de construcción de hipótesis, que serán elemento fundamental en la defensa de sus tesis de grado. En este trabajo argumentamos que la naturaleza de estos errores y obstáculos es ontogenética, psicológica y epistemológica. La naturaleza ontogenética está relacionada con una primacía, conservada por la evolución, de la lógica natural para resolver problemas por sobre la lógica formal. También de las dificultades que acarrea la arbitrariedad del lenguaje. La naturaleza psicológica tiene que ver con una posición del individuo frente a la evidencia y frente a la actividad científica. La evidencia por su contenido corre el riesgo de ser reprimida o rechazada porque constituye una desestabilización de la identidad del sujeto o de las creencias que tienen una función de sostén yoico. Encontramos dependencia psicológica del sujeto hacia sus asesores que les impide construir una afirmación propia. Así mismo, los estudiantes creen que el trabajo académico, más que una empresa de construcción de conocimientos es una actividad evaluadora del yo. Otros errores y obstáculos están relacionados con cuestiones propias de la actividad científica. Una hipótesis, generalmente, manifiesta relaciones causales. La noción de causa y de su naturaleza, desde la filosofía de la ciencia, es debatible. En el presente artículo proponemos algunas explicaciones de las desavenencias en el proceso de construir hipótesis científicas. 
La naturaleza ontogenética, psicológica y epistemológica de los errores y obstáculos en la construcción de hipótesis científicas: un estudio de caso

Palabras clave: hipótesis; discurso argumentativo; errores sistemáticos; obstáculos epistemológicos; tesis de grado; ontogénesis; psicología; ciencia; epistemología; discurso

\begin{abstract}
An analysis of the argumentative discourse of postgraduate students of six different cohorts of the Faculty of Psychology of the National Autonomous University of Mexico, shows us a set of obstacles and systematic errors in the process of construction of a hypothesis (the latter being the hypothesis to defend in their thesis). In this paper, we argue that the nature of these errors and obstacles is ontogenetic, psychological, and epistemological. Ontogenetic obstacles are related to the primacy, reinforced through evolution, of natural logic over formal logic in problem solving. . The arbitrariness of language exacerbates difficulties in communicating a hypothesis. The psychological nature of errors has to do with a position of the individual towards evidence and scientific activity. The content of the evidence may be repressed or rejected because it constitutes a destabilization of the subject's identity or of beliefs that have a function of ego support. We also perceive the subject's epistemic dependence on their thesis advisors that prevents them from affirming their own work. We observe there is a belief that academic work, rather than a process of knowledge building, is an evaluative activity of the self. Other errors and obstacles are related to questions specific to scientific activity. A hypothesis generally manifests causal relationships. The notion of cause and its nature, from the philosophy of science, is debatable. In the present article, we propose these explanations regarding the difficulties in the process of constructing a scientific hypothesis.
\end{abstract}

Keywords: hypothesis; argumentative discourse; systematic errors; epistemological obstacles; thesis; ontogenesis; psychology; science; epistemology; discourse

\title{
Introducción
}

La elaboración de trabajos académicos, como las tesis de grado, los artículos científicos o los proyectos de investigación continúan siendo una exigencia y un requisito de titulación en los programas de posgrado de las principales instituciones en México. Para los estudiantes, cada uno de estos trabajos implica insertarse en la construcción de un discurso argumentativo. Para ello es necesario comprender los elementos básicos de un buen argumento y "ejecutar" el pensamiento 
lógico acerca del pensamiento intuitivo. Para facilitar esta tarea llevamos a cabo durante ocho generaciones de estudiantes de posgrado en Psicología de la Universidad Nacional Autónoma de México (UNAM) y de la Universidad Autónoma del Estado de Morelos (UAEM) un seminario titulado "Seminario de Argumentación Científica", cuyo propósito es acompañar al estudiante para transitar intelectualmente por este camino: el que implica dar un salto cualitativo del pensamiento intuitivo al pensamiento lógico. Para ello se ha utilizado el modelo argumentativo de Toulmin (2003) como heurístico y una didáctica de acompañamiento casi psicoterapéutica. El objetivo de usar el modelo de Toulmin es que los estudiantes construyan el esquema argumental de su investigación con la respectiva presencia de cada uno de los elementos que lo constituyen: datos, hipótesis y/o supuestos, justificaciones, fundamentos y refutadores. Un buen argumento en la ciencia debe contener estos elementos.

El método de acompañamiento que empleamos consiste en apoyar a los estudiantes para ubicar las representaciones mentales acerca de su tema de investigación, de acuerdo con la naturaleza del componente argumentativo del modelo de Toulmin correspondiente, o, en su caso, crearlas. En su trabajo académico la hipótesis tiene especial relevancia. Es la propuesta de conocimiento a probar durante el trabajo de investigación.

En este proceso mental de construcción del esquema argumental hemos identificado un conjunto de errores sistemáticos y obstáculos que dificultan la formulación de hipótesis (o supuestos). Dichos errores también impiden establecer relaciones pertinentes, coherentes, oportunas e inteligibles ${ }^{1}$ entre evidencias e hipótesis. En este trabajo pretendemos defender que la naturaleza de estos errores sistemáticos y obstáculos parece ser ontogenética, psicológica y epistemológica. Nuestro propósito es darlos a conocer y, posteriormente, explicar su naturaleza.

\footnotetext{
${ }^{1}$ Para Vega (2003) la pertinencia de las aserciones consiste en que aquello que se aduce o arguye se refiere a la cuestión o al problema planteado. La coherencia interna se refiere a que entre las razones que integran al argumento deben existir relaciones de implicación materialmente correctas. La oportunidad de las aserciones consiste en que el contenido de los enunciados que integran el argumento proceda de evidencias significativas o de modelos teóricos acreditados por la comunidad frente a la que se argumenta y la inteligibilidad, en que los enunciados que integran el argumento sean objeto de la interpretación más precisa posible, no sean ambiguos, ni vagos, ni confusos.
} 
La naturaleza ontogenética, psicológica y epistemológica de los errores y obstáculos en la construcción de hipótesis científicas: un estudio de caso

\section{Aclaraciones necesarias sobre las hipótesis científicas}

Una hipótesis ${ }^{2}$ o supuesto es un enunciado lingüístico, gráfico o simbólico ${ }^{3}$ que proviene de la acción mental de inferir, la cual consiste en extraer de un conjunto de representaciones que le llamamos evidencias o datos, una que las contiene, pero manifestando nuevas propiedades y posibles relaciones causales entre ellas. Es una acción mental que construye redes teóricas (Lorenzano y Ginnobili, 2015) con relaciones de causalidad entre sus nodos y que tiene intención explicativa. Debido a ello, es una representación más compleja que las evidencias per se, porque es un producto, en un inicio imaginario que une y relaciona elementos de lo perceptible en una representación, a primera vista no perceptible y que mediante los instrumentos de la ciencia pretende probar su veracidad. Una de las tareas del constructor de conocimientos es diseñar los mecanismos que permitan a las otras mentes lograr representaciones similares a las propuestas. ${ }^{4}$

En la investigación científica (siempre que no sea descriptiva o exploratoria) de los investigadores se espera hagan saber sus hipótesis, sobre las que se puede comenzar un debate, una situación de intercambios de representaciones posibles. La hipótesis es uno de los componentes esenciales del discurso argumentativo de la ciencia (Toulmin, 2003; Van Dijk, 1992; Van Dijk, 1998) si está ausente, el discurso del investigador frente a su público parece no tener dirección y en algún momento se le puede cuestionar ¿qué es lo que se desea defender exactamente? o ¿cuál es la propuesta?

Bien sabemos que el conocimiento científico se ha constituido de las propuestas hipotéticas de los investigadores quienes las han hecho explícitas y posteriormente llevan a cabo un conjunto de acciones, lógico-argumentativas y/o metodológicas para constatar su plausibilidad, su verdad o su falsedad ${ }^{5}$.

\footnotetext{
${ }^{2}$ En el presente artículo nos enfocamos en el análisis de hipótesis relevantes, no intuitivas. La relevancia dependerá no sólo de las relaciones de implicación que exista entre ésta y las evidencias disponibles sino en el consenso y la aprobación de su contenido por parte de los expertos involucrados (asesores de tesis, supervisores del caso y colegas). ${ }^{3}$ No omitimos la propuesta de Barceló (2012) acerca de que las imágenes tienen un rol sustancial en la argumentación. Al respecto comenta: "retomando la información del contexto, las imágenes pueden contribuir directa y sustancialmente a la comunicación de las proposiciones que desempeñan el papel de premisas y conclusión. Además, pueden lograr esto directamente, es decir, sin necesidad de verbalización”. p. 355.

${ }^{4}$ Echevarría (1998) argumenta que en la construcción de representaciones científicas (por ejemplo, en la representación de los mapas y su relación con lo mapeado, o en la elaboración de un esquema del ribosoma a partir de una fotografía del ribosoma) más que ser una representación fundada cognitivamente de forma individual, se alude a una representación fundada en una idea sociológica de selección, de prácticas coordinadas por grupos. En las representaciones se introducen elementos que resulten legibles e interpretables desde el marco teórico compartido por la comunidad, en la que se construyen dichas representaciones. pp. 94-95.

${ }^{5}$ Retomamos a Hempel (1973/2003), quien enfatiza en el imprescindible papel de las hipótesis en la actividad científica y cuestiona la concepción inductivista estrecha que ha considerado que esta actividad comienza con la observación.
} 
Es entonces una acción de la mente altamente compleja, que parece necesitar algunas de estas condiciones, 1) estar interesado y curioso $^{6}$ por explicar un fenómeno particular, 2) identificar la evidencia relacionada con el fenómeno e inferir de ella las cualidades que parecen actuar como causa, 3) traducir todas estas representaciones mentales, imaginarias en un enunciado lingüístico (cuya precisión técnica la proporciona el marco teórico de partida), y 4) socializarlo y probar su correspondencia con la realidad que se pretende explicar.

La hipótesis como producto de la habilidad inferencial necesita de la evidencia ${ }^{7}$. Es una construcción que requiere de estos dos elementos, la evidencia y el mecanismo de inferir algo de ellas, que vale la aclaración, es una respuesta potencial a un problema enmarcado en una teoría. De acuerdo con la lógica formal, en la inferencia válida se esperan relaciones de implicación entre la evidencia y la conclusión. Esto se logra formalmente a través del conocimiento y de la aplicación de las reglas de la lógica.

En la labor didáctica que llevamos a cabo con estudiantes de posgrado para la elaboración de su tesis de grado, la evidencia (a la que aludiremos más adelante) apunta a que la tarea de construir una hipótesis no resulta sencilla. Esta condición normativa del pensamiento lógico de lograr una identidad entre nuestras representaciones y formular de ellas otra representación (la hipótesis) parece lidiar con obstáculos de diversa naturaleza: ontogenéticos, psicológicos y epistemológicos.

En la formación de investigadores, construir una hipótesis y contrastarla son acciones necesarias. Una de las pretensiones de los niveles superiores de estudio es garantizar la continuidad en la construcción de conocimientos científicos y una forma de lograrlo es la petición institucional de que los alumnos realicen una tesis de grado. Este es un documento en el que generalmente el

De la misma manera Vega (2003), van Eemereen y Grotendrost (2004) y Perelman y Olbrecht Tyteca (2006) han propuesto una serie de reglas lógicas, dialécticas y persuasivas que intervienen en la determinación de esta veracidad, plausibilidad y falsedad.

${ }^{6}$ Retomamos el valor epistémico que Thagard (2004, 2008), Pérez Ransanz (2005) y De Sousa (2008) otorgan a las emociones como mecanismos psicológicos que actúan en la empresa científica. Desde sus hipótesis, las emociones (entre ellas el interés y la curiosidad) cumplen funciones selectivas y heurísticas. Participan en los procesos de evaluación de evidencias, en la selección de las que parecen ser relevantes para la explicación del fenómeno y descartando las que no lo son.

${ }^{7}$ En Hempel (1988), las inferencias hipotéticas deben partir fundamentalmente, de premisas con contenido empírico, es decir que por lo menos en principio, sea posible comprobarlo mediante el experimento y la observación. Sin embargo, considera que la inferencia no sólo se apoya en evidencias "brutas" o directamente perceptibles, sino también en otros hechos "no directamente perceptibles" pero que dan lugar a la expresión fenomenológica de lo que se pretende explicar. A las premisas las acompañan también un conjunto de leyes generales, teóricas o regularidades que son indispensables para la comprensión de la inferencia.

$\mathrm{N}^{\mathrm{o}}$ 26, Vol. 13 (2), 2021. ISSN 2007 - 0705, pp.: 1 - 24

- 5 - 
La naturaleza ontogenética, psicológica y epistemológica de los errores y obstáculos en la construcción de hipótesis científicas: un estudio de caso

estudiante se encuentra ante la situación de proponer una hipótesis o un supuesto y diseñar un procedimiento para probarla.

Es en esta tarea donde encontramos los obstáculos. Bachelard, 1948/2003 propone una secuencia evolutiva en la historia de la ciencia en la que los nuevos conceptos se construyen a partir de la superación de conceptos anteriores, que por una parte constituyen obstáculos para ese desarrollo, pero por el otro son condición de posibilidad de esa superación. A partir de la noción original de Bachelard, Brousseau, 2003, caracteriza a los obstáculos epistemológicos en los contextos de enseñanza y aprendizaje como concepciones o "conocimientos" parciales e incompletos, estables y difíciles de remover, que resultan pertinentes para la resolución de problemas dentro de ciertos contextos limitados, pero que muestran limitaciones o conducen a errores si se intenta generalizar su aplicación. En tanto tales, también cumplen la doble función de ser punto de partida, y a la vez constituir obstáculos que dificultan la generación o adquisición de nuevos conocimientos o conceptos más generales. Asimismo se presenta un conjunto de errores sistemáticos, tal como los entienden Palau y Couló (2001, cf. infra) que dan cuenta de la presencia de tales obstáculos epistemológicos en la resolución de la tarea por parte de los estudiantes.]

\section{Los obstáculos y errores sistemáticos en la construcción de hipótesis de investigación: un estudio de caso \\ Método}

Antes de dar a conocer los obstáculos y errores comenzaremos por contextualizar el ámbito de nuestra investigación. Debido a la baja eficiencia de titulación de uno de los programas de posgrado de la Facultad de Psicología de la UNAM se creó un curso de dos semestres para apoyar a los estudiantes para la elaboración y conclusión de su tesis de grado. Como estrategia didáctica utilizamos el modelo argumentativo de Stephen Toulmin como un heurístico ${ }^{8}$ mental. El curso comenzó en el año 2011 y continúa hasta la fecha. Hemos trabajado con un total de 77 estudiantes (aproximadamente 13 alumnos por generación, 64\% son mujeres y la media de edad es de 28 años). Para obtener el grado se les solicita un reporte de experiencia profesional cuya temática es el

\footnotetext{
${ }^{8}$ Los heurísticos son considerados mecanismos, instrucciones o reglas mentales que permiten solucionar problemas específicos y hacen más eficientes los procesos cognitivos, sobre todo aquellos que parecen ser computacionalmente costosos. No necesariamente llevan a soluciones correctas o son garantías de obtener la solución, pero es como racionalmente actuamos (Eraña, 2003): "son sistemas de instrucciones que nos permiten solucionar un problema, no de manera exacta ni en todos los casos, pero sí de manera que no sea necesaria una computación excesiva de los recursos cognitivos que tenemos disponibles" (citado en Stincer y Blum, 2017).
} 
análisis de un caso clínico (de un adolescente). También se les permite realizar investigaciones documentales e investigaciones empíricas de corte cuantitativo. 96.1\% ha elegido la opción del estudio de caso clínico. Es importante aclarar que utilizamos el modelo de Toulmin para apoyar la construcción del discurso escrito y oral de la tesis. A esta construcción le antecede un proceso de investigación (de aproximadamente un año) que les permite contar con evidencia "suficiente" acerca del caso clínico para poder elaborar una hipótesis.

El conocimiento de los obstáculos y errores sistemáticos a los que hacemos referencia lo inferimos del análisis del discurso más recurrente de los estudiantes al formular su hipótesis. Siendo así, este trabajo se apoya en la modalidad cualitativa de investigación y en la estrategia del estudio de casos.

Partimos del supuesto de que la tesis es un documento que requiere apoyarse en los elementos de un discurso argumentativo y el modelo de Toulmin explica los componentes básicos de un argumento: 1) los datos (data), 2) la conclusión (claims), 3) las justificaciones (warrants), 4) los fundamentos o respaldos (backing), 5) los refutadores (rebbutals) y 6) los calificadores modales (qualifiers). En la Fig. 1 se muestra el modelo con los componentes mencionados, la estructura y el orden secuencial que propone Toulmin debe existir entre ellos.

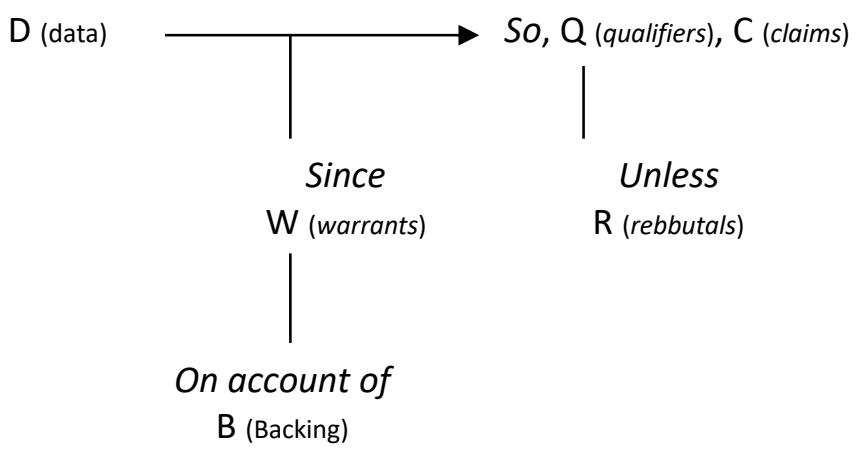

Fig. 1. Esquema del modelo de Toulmin: extraído de Toulmin, S. (1958/2003). The uses of argument. Cambridge University Press.

Fig. 1. Schematic of the Toulmin model: extraído de Toulmin, S. (1958/2003). The uses of argument. Cambridge University Press.

Inicialmente, los estudiantes precisan el objeto de su investigación y comprenden la estructura del modelo de Toulmin. La propuesta didáctica consiste en que comience la identificación y ubicación de las representaciones mentales respecto al tema, de acuerdo con la naturaleza del componente correspondiente. Los datos o evidencias en su lugar, la hipótesis en el suyo (si no existe, se 
La naturaleza ontogenética, psicológica y epistemológica de los errores y obstáculos en la construcción de hipótesis científicas: un estudio de caso

construye), y así para todos los componentes. Para ello se utiliza una simple tabla (ver Fig. 2) en la que escriben sus representaciones (Stincer y Blum, 2017).

Fig. 2. Tabla de apoyo para estudiantes con los componentes del modelo de Toulmin.

Fig. 2. Student support table with the components of the Toulmin model.

\begin{tabular}{|c|c|}
\hline Evidencias & Hipótesis o supuesto \\
\hline & \\
\hline & Justificaciones \\
\hline \multicolumn{2}{|c|}{ Fundamentos o respaldos } \\
\hline \multicolumn{2}{|c|}{ Refutadores } \\
\hline
\end{tabular}

Nota: Tabla en formato Word en la que los estudiantes escriben el contenido de sus representaciones de acuerdo con la naturaleza del componente argumentativo abordado.

Note: Word format table in which students write the content of their representations according to the nature of the argumentative component in question.

La formulación de cada apartado se realiza individualmente y se somete a debate grupal. El contenido pasa por el escrutinio reflexivo del grupo, quienes comparten un marco conceptual común (el programa de maestría en cuestión se apoya fundamentalmente en la teoría psicoanalítica para el análisis de casos clínicos). Este escrutinio tiende a proporcionar elementos válidos para afinar cada una de las partes.

La experiencia con seis generaciones de estudiantes ha proporcionado evidencia de que las mayores dificultades aparecen al llenar los dos primeros recuadros de la tabla (hacer explícitas las evidencias y construir la hipótesis que "dé cuenta" de ellas). Esto significa lograr relaciones de implicación entre la evidencia y la hipótesis, y que se respete la forma lógica $P \rightarrow Q$ entendida como la implicación condicional de un argumento. Además, resulta más complejo porque esta actividad es esencialmente un proceso inferencial abductivo: dentro de un conjunto de inferencias posibles debe elegirse la que mejor explique las evidencias disponibles. Es en ese momento en el que aparecen los obstáculos y los errores sistemáticos.

Para entenderlos como obstáculos retomamos el concepto de Bachelard (1948/2003) que los considera como condiciones psicológicas y epistemológicas que funcionan como causas de estancamiento, de retroceso y de inercia en los procesos de razonamiento. Los errores sistemáticos, de acuerdo con Palau y Couló (2011), son los productos perceptibles de las consecuencias de estos obstáculos en el razonamiento lógico. Se manifiestan como textos (conjunto de enunciados), acciones e imágenes que aparecen con frecuencia. Pueden ser predecibles, no son productos de descuidos ni falta de atención, tienen coherencia, es decir, no son enunciados aislados, sino que aparecen en conexión con otros o interconectados en redes y tienden a ser recurrentes, tanto 
individual como grupalmente (Charnay y Mante, 1992 citados por Palau y Couló, 2011, p. 184). Algunos ejemplos de errores sistemáticos encontrados, son por ejemplo el hecho de que los estudiantes rechazan el principio de que una conclusión verdadera puede ser inferida de premisas falsas desde un esquema de razonamiento válido, o bien la dificultad para aceptar que cualquier evidencia disponible es siempre insuficiente para garantizar la verdad de las hipótesis planteadas, estos pueden atribuirse a una serie de obstáculos tales como 1) la lógica natural no construye series de inferencias sintácticamente complejas como las de la lógica formal, 2) los argumentos de la lógica natural son difíciles de analizar porque involucran una dimensión pragmática, 3) los argumentos de la lógica natural no siguen una secuencia inferencial paso a paso, más bien tienen saltos inferenciales "agigantados" por falta de información o por presuposiciones, 4) las inferencias de la lógica natural suelen estar vinculadas a las creencias de los hablantes, son contextodependientes."

Los errores sistemáticos vinculados a los obstáculos no son aleatorios, implican una construcción incompleta, insuficiente, pero al mismo tiempo un paso en la formación de un conocimiento más adecuado.

En el curso de posgrado mencionado, la tesis de grado es el estudio de un caso clínico con más de 20 sesiones psicoterapéuticas de trabajo, por tanto, se cuenta con evidencias que permitirían elaborar una hipótesis acerca del caso.

Un análisis del discurso emitido por los estudiantes al construir la hipótesis revela que los obstáculos y los errores sistemáticos más frecuentes son los siguientes:

1) Incluso contando con 20 o más sesiones terapéuticas, los estudiantes consideran que las evidencias con las que cuentan son insuficientes para inferir "algo".

Al respecto mencionan:

E1: Profesora, aunque llevo con el caso 43 sesiones, no me atrevería a afirmar nada sobre él. Es un caso muy complejo, con muchas aristas, puede verse desde diversos lados y si elijo una, descuido las demás. Eso no me gusta, ¿qué tal si afirmo algo y es otra cosa?

E2: Yo llevo con el caso 35 sesiones, pero tengo los datos del caso hasta aquí, hasta el momento actual, pero mañana, en la consulta, pueden aparecer nuevos datos y mi hipótesis irse al piso.

2) Contrario a lo anterior, los estudiantes logran inferir, pero no hacen explícitas la mayoría de las evidencias que permiten comprender su inferencia. Las evidencias que reportan provienen 
más de la intuición que de los hechos. Construyen la hipótesis a partir de una creencia o experiencia afectiva que les informa que "algo debe haber ahí y aunque no lo han visto, está".

E3: Tengo un caso que funciona psicóticamente porque la familia se beneficia de su psicosis.

Ante la petición del docente de hacer explícitas las evidencias que le permiten inferir esto el estudiante contesta:

E4: Nunca he podido ver a la familia, pero es la impresión que tengo, "me late" que la familia parece funcionar bien gracias a la psicosis del caso.

3) La hipótesis es considerada por los estudiantes como un enunciado que marca una posición absolutista, "cerrada", que suprime la riqueza y la complejidad de cualquier caso, por lo que hay estudiantes que prefieren no formularla o hacerla explícita.

E5: Profesora, ¿es obligado plantear una hipótesis?, eso es aferrarse a una sola idea, a una sola explicación, a mí me angustia cerrarme a una sola mirada del caso, yo preferiría sostener varias hipótesis o no comprometerme con ninguna.

E6: Profesora, la hipótesis quedará muy extensa porque son muchas las causas que pueden estar determinando la problemática del caso... si nos basamos en la teoría de la complejidad peor aún, hasta el aleteo de una mariposa puede ser. Es mejor no comprometernos con una sola idea.

4) Algunos estudiantes consideran "atrevida" la posibilidad de inferir, de extraer algo de las evidencias.

E7: Profesora, es que me resulta difícil afirmar algo, ¿yo puedo?, ¿no es muy subjetivo?, ¿no están para eso los autores?, ¿no se supone que yo debo retomar lo que ellos dicen?

E8: Yo prefiero preguntarle a mi asesor, que él sea el que me diga la hipótesis, él tiene más experiencia que yo.

E9: Construí la hipótesis, pero a mi asesor no le pareció bien y me propuso "ésta" y yo me quedaré con ella.

5) Un error sistemático es el siguiente: al debatir la propuesta hipotética de uno de los estudiantes, sobre todo de los primeros, algunos compañeros tienden a no valorar la evidencia presentada y demandan otras evidencias a las que no se les está dando relevancia. Es decir, intentan desviar la "mirada" de esa persona hacia la mirada de ellos, buscan persuadir para que asuman otra inferencia. Tienden a no respetar la inferencia ni las evidencias proporcionadas. 
6) Ante la solicitud de que en las hipótesis se perciban las relaciones causales, algunos estudiantes consideran que la noción de causalidad es cuestionable. Para algunos las causas no existen, adoptan concepciones humanas, y plantean que ellas son una construcción de la mente y no un tipo de relación que pueda constatarse. Siendo así, las hipótesis se construyen sobre "relaciones posiblemente falsas".

E10: Profesora, la hipótesis nos obliga a comprometernos con una, dos o " $n$ " causas para un determinado problema y sabemos que en el análisis de un caso lo que hoy consideramos la posible causa de la sintomatología, mañana puede no serlo o no ser la única. Entonces nuestra tesis se cierra a un conjunto de causas que pueden explicar el problema en un determinado momento y en otro no. Con las hipótesis se nos escapa la posibilidad de abordar el problema desde toda su complejidad. Eso sin entrar en la discusión de la existencia de causas y relaciones determinísticas entre las cosas. Hay filósofos que dudan de este concepto, lo consideran una construcción de la mente, no una realidad exterior. Es un deseo nuestro establecer relaciones de dependencia entre una cosa y otra, ¿cómo sostener una hipótesis con estas condiciones?

Llama la atención cómo en el proceso inferencial de construir una hipótesis aparecen estos obstáculos y errores que dan cuenta de la gran dificultad que trae consigo la estructura lógica $p$ implica q. Desde la dimensión lógica de la argumentación, Copi (1979), Reñón (2003), Beuchot (2008), entre otros, consideran que un buen argumento ${ }^{9}$ es un conjunto de enunciados en los que unos son premisas y otro la conclusión, y la última debe estar contenida en las primeras. Las relaciones de implicación entre ellas deben ser válidas y este atributo, en la lógica formal, lo determina el apego de la estructura de los enunciados a las condiciones establecidas en las tablas de verdad y el respeto a las reglas lógicas. En nuestro curso, las relaciones de implicación entre evidencias e hipótesis se discuten sobre la percepción de relaciones plausibles ${ }^{10}$ entre el contenido de las evidencias y la representación abstracta generada por ellas, intentando apegarnos a las

\footnotetext{
${ }^{9}$ Hacemos hincapié en que es en la dimensión lógica de la teoría de la argumentación donde un buen argumento es sólo aquel que es formalmente válido, es decir, que se apega a las estructuras de las reglas lógicas. En las dimensiones dialécticas y retóricas consideran "buenos argumentos" aquellas propuestas que son aceptadas (de forma regulada y consensuada) por los miembros inmersos en una situación argumentativa o aquel argumento que persuade, aunque sean formalmente inválidos.

${ }^{10}$ Comprometidos, un poco más, con las dimensiones dialécticas y retóricas de la teoría de la argumentación, sin dejar de perseguir el ideal de un buen argumento de acuerdo con Vega (2003), quien plantea que éste debería ser formalmente válido, plausible y convincente.
} 
La naturaleza ontogenética, psicológica y epistemológica de los errores y obstáculos en la construcción de hipótesis científicas: un estudio de caso

condiciones y reglas lógicas. Las relaciones establecidas por ellos deben reflejar vínculos causales posibles que sean consistentes con la teoría de partida.

Es este discurrir de la mente en la elaboración de hipótesis, presentado por nuestros estudiantes, lo que consideramos obstáculos ontogenéticos, epistemológicos y psicológicos.

\section{Los obstáculos ontogenéticos en la construcción de hipótesis científicas}

Desde una perspectiva racionalista y representacional, la hipótesis es un enunciado que proviene de la razón, de su posibilidad de dar sentido y significado a las evidencias presentes (van Fraassen (1980), Linch y Woolgard (1990) Giere, (1992), Hacking (1993), Ibarra y Mormann (1997). Es una de las maneras en que estas representaciones racionales salen a la luz y lo hacen generalmente en forma de enunciado verbal. En la actividad no científica pueden permanecer como una creencia o un punto de vista, pero en la actividad científica es necesario probar la hipótesis.

La construcción de la hipótesis parece ser una competencia cognitiva que "nos está dada", un producto de la ontogenia. Un mecanismo mental conservado por la evolución que permite que el hombre, como especie, interactúe con el mundo, lo conozca, funcione en él, y saque conclusiones de lo que ocurre. Sin embargo, es una competencia cognitiva que parece tener dos modalidades de actuación: una intuitiva (natural) y otra analítica (sofisticada y formal) ${ }^{11}$. La última entra en juego cuando para la especie es importante distinguir entre lo cierto y lo falso, lo que es y lo que no. Ante este problema, la competencia requiere de la modalidad sofisticada pero interviene la natural. Esta intervención es producto de la ontogenia, es constitutivo del funcionamiento cognitivo ${ }^{12}$. La

\footnotetext{
${ }^{11}$ Las diferencias entre una y otra modalidad está dada por la estructura y el contenido de los elementos que componen las explicaciones de hechos que cada una de ellas genera. Estas explicaciones están conformadas por premisas y conclusiones (explanandum y el explanans). Tradicionalmente, en las explicaciones científicas, siguiendo a Nagel (1981) el sujeto asume y hace explícitas ciertas condiciones que deben cumplir las premisas para poder producir una conclusión. Dependiendo del dominio donde se encuentre el hecho que se esté explicando 1) las premisas deben tener un carácter necesario para determinar el valor de verdad de las conclusiones, 2) las premisas deben basarse en juicios observacionales y experimentales, 3 ) deben ser una regularidad estadística (no sólo estadística, pueden expresar una regularidad universal), 4) hacer referencia explícita a un estado o suceso futuro que hace inteligible la existencia de la cosa explicada y 5) en algunos casos particulares, se debe conocer el gran número de enunciados singulares relativos a acontecimientos pasados que le dieron lugar a las premisas y reconocer que entre estos acontecimientos hay dependencias causales. Como dijera Hempel (1988), es necesario establecer las condiciones iniciales en las que el fenómeno se da, estas pueden ser pragmáticas o teóricas. En la modalidad del sentido común, esta tarea no se lleva a cabo, se deja al arbitrio, por lo tanto, las relaciones entre explanandum y explanans no son precisas, ni sistemáticas ni adecuadamente fundamentadas.

12 "No deben considerarse como mera falta de conocimiento, sino como un paso en la construcción del mismo, un importante punto de partida en el desarrollo de nuestra comprensión y entendimiento" (Palau y Couló, 2011, p. 186).
}

№ 26, Vol. 13 (2), 2021. ISSN 2007 - 0705, pp.: $1-24$

- 12 - 
evolución, retomando a De Sousa (2008), ha dejado una desafortunada mezcla de ambas modalidades para obtener conocimientos verdaderos o plausibles.

Todos podemos construir una hipótesis y "lo ideal" es hacerlo con claros y meticulosos principios y reglas lógicas, sin embargo, la evidencia apunta a que así no funciona la competencia inferencial (Eraña, 2003). Esta parece apoyarse en principios menos meticulosos, borrosos y difusos propios de lo que se conoce como lógica natural o lógica folk (Palau, 2014; Monroy, 2017; Couló, 2017). La intervención de esta lógica natural aparece como un obstáculo para la construcción de razonamientos y argumentos lógicos.

La distinción entre lógica formal y lógica natural es que la primera es una abstracción de las estructuras que parecen regir los pensamientos correctos o aquellos que logran mostrar relaciones de implicación válidas entre premisas y conclusiones. Son independientes del contenido que se le atribuya; se cumplen en cualquier mundo posible, por tanto, no son dependientes del contexto. Para Palau (2014), la lógica natural se forma por las acciones mismas y por el pensamiento espontáneo. Los enunciados que provienen de ella involucran una dimensión pragmática, relacionada con lo inmediatamente perceptible, con lo que funciona para la supervivencia y con la comunicación interpersonal directa (con sus usos y supuestos compartidos, con sus connotaciones, etc.). En su estructura se aprecian grandes saltos inferenciales que reflejan falta de información, presuposiciones no explicitadas, intuiciones, estados o representaciones que, de acuerdo con Pérez Ransanz (2005) y Hookway (2008), pueden ser impulsadas por emociones y no necesariamente serían incorrectos porque éstas portan contenido relevante acerca de los fenómenos, pero no han sido traducidos o computados verbalmente o mediante una representación que pueda ser entendida. En la lógica natural, las inferencias de los hablantes están ligadas a sus creencias, son contexto-dependientes, no toleran la descontextualización.

Siendo así, los errores sistemáticos de los razonamientos involucrados en la construcción de hipótesis no aparecen "por gusto", son producto de una constitución ontogenética con una marcada orientación pragmática. La lógica natural aparece impidiendo el despliegue fluido de la lógica formal, aunque Palau (2014) sostiene que esta última depende de la primera y Barceló (2005) sugiere que el lenguaje formal de la lógica se aprende desde el natural y es un error didáctico presentar el lenguaje lógico como ideal o una sofisticación y depuración del natural.

El error de constitución ontogenético, sugerimos una vez más, está en tener la existencia de estos dos sectores de la mente, como sostiene De Sousa (2008) "The two track mind": el intuitivo 
La naturaleza ontogenética, psicológica y epistemológica de los errores y obstáculos en la construcción de hipótesis científicas: un estudio de caso

y el analítico. El primero permite funcionar en un nivel básico, el segundo descubre relaciones complejas y ocultas a la percepción del mundo, pero es computacionalmente más costoso. El primer sector provee de contenido al segundo y el segundo requiere de abstraerse de lo directamente perceptible para develar las posibles regularidades de ello. La evolución no permitió una primacía e independencia del pensamiento lógico formal, es vasta la evidencia, de que la mayor parte de las personas no nos apoyamos en los principios lógicos ${ }^{13}$, lo que incrementa la probabilidad de cometer errores y creer en acontecimientos falsos. Lo consideraremos un error de constitución porque resulta que un pensamiento apoyado en los principios de la lógica formal, como afirma Barceló (2005):

1) Nos ayuda a pensar y razonar mejor ${ }^{14}$.

2) Nos enseña a conocer y a usar el lenguaje para interpretar, construir y evaluar argumentos.

3) Nos permite describir cosas que se dan de hecho, como qué sigue a qué, o qué proposiciones son lógicamente verdaderas.

4) Pedir y dar razones de lo que hacemos y creemos (las "prácticas inferenciales"). Estudiamos lógica para aprender qué debemos hacer para convencer y llegar a acuerdos racionales.

Sin embargo, no parece ser así. La lógica formal no emerge espontáneamente, requiere de la educación, la didáctica y de un ejercicio intelectual concienzudo. Con ello no se niega que algunos seres poseen, naturalmente, un predominio del pensar desde este sistema analítico de la mente ${ }^{15}$. Pero es un sistema que generalmente requiere instrucción. Por ello, una de las preocupaciones fundamentales de Monroy (2017) ante la dificultad de la enseñanza y el aprendizaje de la Lógica es que, equívocamente, se solucione eliminándola de los planes de estudio en el nivel medio superior y superior. Con ello se obstruye la posibilidad de fortalecer este sistema y por ende se arraiga el sistema intuitivo: funcional (básico y pobre).

A favor de esta hipótesis del error de constitución ontogenético están los hallazgos de las investigaciones en neurociencias que han constatado experimentalmente que sesgos del

\footnotetext{
${ }^{13} \mathrm{Al}$ menos desde las investigaciones de Wason (1960), se ha trabajado sobre los errores y sesgos cognitivos. Algunos autores proponen considerar esto como una evidencia de las constricciones que la constitución biológica y psicológica de los seres humanos plantea a la racionalidad (bounded rationality).

${ }^{14}$ Apoyados en las reglas y principios lógicos que de forma sistemática han conducido a conocimientos verdaderos o plausibles (Vega, 2003).

${ }^{15}$ Lo podemos inferir de los resultados experimentales de Evans (1989) sobre los errores del razonamiento lógico en el que constata que más de $90 \%$ de individuos que realizan un test en el que se propone una tarea de refutación de reglas condicionales, se equivoca. Hay un porcentaje pequeño (menos de $90 \%$ ) que no se equivoca. Puede pensarse que ellos son un ejemplo del uso "natural" del sistema analítico por encima del intuitivo.
} 
razonamiento tienen su base cerebral. Houde, Moutier, Zago y Tzourio-Mazoyer (2003) en su artículo "La realidad biológica de la irracionalidad" dan a conocer la ubicación de las redes neuronales de estos errores. En sus experimentos, constatan que más de $90 \%$ de los sujetos al realizar una tarea lógica de refutación de condicionales ${ }^{16}$ no la resuelve satisfactoriamente porque en lugar de tomar en cuenta las condiciones lógicas de los enunciados o las reglas, los participantes se concentran en la percepción de los elementos citados en la regla. Para la respuesta correcta era necesario deshacerse de los elementos perceptibles en los enunciados. A esto Evan (citado por Houde y colaboradores, 2003) le denominó "sesgo de emparejamiento perceptivo", el adulto "se deja engañar" por la percepción y olvida las consignas lógicas. En esta tarea, como plantean Houde y colaboradores (2003), la respuesta correcta requería ir contra de la percepción de los elementos citados en la regla, contra el emparejamiento perceptivo. Los autores consideran que en el interior del espacio mental entran en conflicto dos estrategias: la perceptiva y la lógica. El sujeto no inhibe la estrategia perceptiva, y ésta le toma la delantera a la lógica. Ellos sugieren que son necesarios mecanismos de bloqueo de estas estrategias y han diseñado un conjunto de ellas: los inhibidores de las estrategias perceptivas. Con estos inhibidores, la cifra de sujetos que comete el error del emparejamiento perceptivo disminuye considerablemente, dando lugar a una aplicación efectiva de la estrategia lógica. Esto es didácticamente muy interesante.

Lo importante de estos estudios es quizás la confirmación de que la lógica natural es aún más natural de lo imaginado, al sostenerse en redes cerebrales que priorizan lo inmediatamente perceptible sobre lo lógico ${ }^{17}$.

\section{Las cuestiones psicológicas y epistemológicas que irrumpen en la construcción de hipótesis}

Si releemos los discursos de los estudiantes también podemos vislumbrar obstáculos de naturaleza psicológica y epistemológica como estos: 1) el predominio del afecto de la incertidumbre, 2) la sobreestimación de las intuiciones, 3) la dependencia psicológica a la autoridad, 3) la presencia de

\footnotetext{
${ }^{16}$ La tarea consiste en identificar en un conjunto de figuras geométricas con diversos colores las combinaciones que señalaran que la regla "si no hay un cuadrado rojo a la izquierda, entonces hay un círculo amarillo a la derecha" era falsa.

${ }^{17}$ Los errores ontogenéticos de constitución de lo mental, tiene su equivalente en los errores de constitución física. Olshanki, Carnes y Butler (2001) consideran que el diseño evolutivo de nuestro físico que se ha sostenido biológicamente hasta la actualidad tiene errores o partes mal diseñadas y ello explica ciertas dolencias y el deterioro progresivo del cuerpo con la edad. Ellos comentan que un diseño físico de otro tipo (el cual proponen) nos hubiera permitido un envejecimiento menos doloroso y de menos deterioro progresivo que el que vivimos.
} 
un yo perseguido o evaluado, y 4) el predominio de creencias epistemológicas "positivistas" acerca de la actividad científica.

El punto 1 lo apreciamos cuando los estudiantes consideran que las evidencias para elaborar su hipótesis de trabajo son infinitas o siempre insuficientes. Recordemos que son estudiantes de posgrado y para su trabajo de tesis les solicitamos proponer una hipótesis respecto de un caso con el cual llevan más de 20 sesiones terapéuticas. Al contar con este conjunto de evidencias, considerar que son insuficientes nos hace pensar que (retomando a los epistemólogos de la emoción como Thagard, 2008; De Sousa, 2008; Hookway, 2008; Elgin, 2008; Brun, Doğuoğlu y Kuenzle, 2008) sus procesos racionales están siendo obstaculizados por el afecto de la incertidumbre. De Sousa (2008), en su artículo "Epistemic feeling", propone que en los procesos de deliberación racional, donde es necesario elegir entre diversas alternativas, las que tienen una plausible relación causal con el fenómeno de interés y las que no, es necesario que se ponga en marcha un mecanismo que permita hacer esta distinción y elección. Desde Damasio (2007), y tomando como referente a los sujetos con daños prefrontales cuya principal área cerebral afectada es la emocional y cuyo daño impide elegir y frenar los procesos de deliberación racional, el mecanismo detectado es de naturaleza emocional. Ante ello, De Sousa (2008) menciona la certeza y la incertidumbre como afectos para distinguir y saber elegir entre diversas alternativas. Ante una multiplicidad de evidencias, la certeza proporciona contenido acerca de las que deben ser elegidas y la incertidumbre impediría seleccionar las que parecen no ser relevantes o desechar las que no lo son. Ambas parecen tener un gran valor epistémico e intuimos que lo que determina su función epistémica relevante o no es cuantitativo. Un predominio (en cantidad) de una de ellas tendría consecuencias negativas para el proceso racional porque el contenido que aporta es arbitrario. En el caso de los estudiantes, la dosis de la emoción de la incertidumbre parece ser alta, ello le impide reconocer el valor o la relevancia de la evidencia disponible y extraer de ella una propuesta interesante de conocimiento. El proceso inferencial está siendo afectado por la cantidad existente de esta emoción (o de sus componentes moleculares) en el sujeto (ver extractos E1, E5, E6 y E7).

Pasamos a otro de los errores sistemáticos presente en los comentarios de los estudiantes, aquel que consiste en construir inferencias sin evidencias explícitas. Además de la intervención de la lógica natural parecen estar actuando tres mecanismos psicológicos (que se constituyen en errores). El primero de ellos, y retomando a la teoría psicoanalítica, parece suscitarlo la represión: la evidencia sí existe pero ellos no la hacen explícita porque no está elaborada en su nivel consciente 
o, como indican los psicoanalistas, metabolizada en este plano: "apalabrada". Y no lo está por alguna resistencia a ella vinculada con la propia historia de vida.

El segundo error proviene del deseo interno de que la evidencia sí exista para poder sostener la explicación del fenómeno que resulta inquietante. Este error sí debería sucumbir a la desaparición de la inferencia porque es una evidencia deseada, no existente. Un tercer error se debe a la presencia de "intuiciones", que son un conjunto de experiencias afectivas que portan información proveniente de otros casos y de la experiencia misma pero no precisamente de la evidencia disponible. Esto hace pensar al sujeto que, aunque no haya tenido acceso a la evidencia, es probable que exista y le permitiría comprobar que su inferencia es correcta. Es la propuesta del pensamiento heurístico de Kahneman (2012): aquellas estrategias que nos ayudan a encontrar respuestas a menudo imperfectas a preguntas difíciles y tomar decisiones en entornos de incertidumbre (citado por Garrido, 2013) o de datos para el éxito, son insuficientes.

Otra de las razones de naturaleza psicológica que parece afectar la percepción adecuada de las evidencias, y con ello el logro de una inferencia lógica, es propuesta por la filósofa Ángeles Eraña $^{18}$. De acuerdo con ella, los sujetos se resisten a la evidencia para preservar su identidad. Esto es muy interesante porque desde su punto de vista la identidad del sujeto se sostiene en un conjunto de creencias acerca del mundo y de sí mismos, lo que le permite funcionar con cierta estabilidad. Estas creencias, plantea Eraña, "se atrincheran”, se convierten en el sostén de las acciones del sujeto, en un sostén yoico. La evidencia o dato que se oponga a ellas puede no ser percibida adecuadamente o ser rechazada. Aceptar ciertas evidencias implicaría ir contra estas ideas "atrincheradas" y sostén de nuestro ser, y poner a "tambalear" nuestra estabilidad yoica. Ello explicaría por qué los sujetos se resisten a ellas, "o no las quieren ver". Asumirlas implicaría una lucha interna, quizás angustiante, que pondría en riesgo lo que se cree de sí mismo y del mundo. Se asocia un poco con las ideas iniciales del término "cambio conceptual" (Posner, Strike, Hewson, y Gertzog, 1982) considerado "casi" como una catarsis intelectual mediante la que los sujetos tenían que cambiar los supuestos propios por otros inconmensurables y por ello experimentaban angustia y sufrimiento, porque implicaba cambiar lo que "hasta ese momento para ellos" era "cierto" o "plausible".

\footnotetext{
${ }^{18}$ Contenido extraído de su ponencia "La naturaleza de las creencias: verdad e identidad" expuesta en el Coloquio: Explicación, Creencia y Conocimiento en la Filosofía de la Ciencia, en la Facultad de Psicología de la Universidad Nacional Autónoma de México el 26 de octubre de 2017.
} 
Otra de las dificultades que vislumbramos en el contenido de los discursos para la construcción de una hipótesis parece provenir de esta creencia: "una hipótesis es la afirmación de una verdad y eso es demasiado comprometedor". En general, para los estudiantes en proceso de formación como investigadores, el trabajo de tesis es un proceso de decir verdades, un proceso de evaluación de sí mismos, donde el yo y la identidad son juzgados en su potencial intelectual, no es percibido como un proceso de construcción de conocimientos. Esta creencia les impide proponer "algo", ser cuestionados, otorgar la fuerza de la evidencia de la que disponen (defenderla), considerar el cuestionamiento como algo favorable para el proceso de construcción del conocimiento, donde el amor por sí mismos no está siendo perseguido. Ello provoca que desvíen la actividad mental de un propósito epistemológico hacia uno psicológico que denota dependencia a cuestiones no epistémicas. No afirman para no ser atacados, les es preferible asumir el punto de vista del asesor que el propio, así la responsabilidad del error recae sobre éste y no sobre sí mismos. Les hace sobreestimar el punto de vista del otro, incluso cuando este último no dispone de evidencia, como él.

Otra cuestión que obstaculiza la posibilidad de construir una hipótesis es el compromiso de algunos estudiantes con posiciones asociadas a la noción más ingenua del relativismo epistemológico. Aquel que "hace referencia al sistema o doctrina que sostiene que todos los puntos de vista sobre una misma cuestión son igualmente válidos o correctos (o inválidos e incorrectos)" (Guillaumin, 2009, p. 141). De acuerdo con Kuhn, Cheney y Weinstock (2000) estas se manifiestan con mayor notoriedad en la etapa adolescente. Desde estas posiciones, debido a la cantidad de alternativas, de explicaciones posibles, y por la falta de un mecanismo evaluador que permita elegir la mejor opción, para ellos "o todo es posible como explicación o no lo es".

Otras posiciones epistemológicas son más absolutistas (Kuhn y colaboradores, 2000) y ello lo podemos notar en los cuestionamientos de los estudiantes acerca de la noción de causalidad. Se aprecia un compromiso más fuerte hacia tal noción que proporcionan las ciencias naturales, la cual apunta a regularidades o leyes universales relativamente estables y donde las causas tienden a comportarse de manera determinista que, con la noción de causa histórica, la cual tiene un papel determinante para la ocurrencia de un evento social pero no lo será para otro porque fue contextual y única. Esto impide la creación de una red teórica con poder explicativo (ver como ejemplos los extractos E6 y E9). 


\section{El lenguaje y sus arbitrariedades con el pensamiento}

En la comprensión de este problema también percibimos que influye la dinámica entre el pensamiento y el lenguaje. No se discutirá si uno depende del otro o si son funciones independientes entre sí. No obstante, coincidimos con Vosgerau (2012) respecto a que recurrimos al lenguaje para expresar nuestras ideas, y que en ocasiones "damos muchas vueltas a las palabras" sin dar con la formulación correcta. Sabemos lo que pensamos y lo que nos gustaría expresar, pero nos resulta difícil expresarlo (especialmente por escrito) y experimentamos explicaciones verbales inútiles, ello indica las dificultades del lenguaje para traducir lo representado en el pensamiento. También compartimos con este autor la idea de que al pensamiento lo constituyen conceptos (términos lingüísticos) que se refieren a objetos, y que en el caso particular de conceptos teóricos se requiere una comprensión de la teoría completa y su terminología. Así, la formulación de una hipótesis científica es dependiente del lenguaje. Cuando leemos una hipótesis lo que vemos es una oración, no el pensamiento en si $^{19}$.

Saussure (1945) nos alertó sobre la arbitrariedad de los signos lingüísticos respecto a lo que pretenden designar. Las palabras guardan estrecha o nula relación con la cosa designada. De manera que las ansiedades y dificultades de una persona para construir una hipótesis pueden deberse a la incapacidad para representar "con fidelidad", a través del lenguaje, la representación mental que se desea designar. En ocasiones nos percatamos de que el estudiante lleva un proceso mental lógico serio, sin embargo, le resulta complejo encontrar el concepto o término lingüístico adecuado que represente (como él desea) el fenómeno objeto de su representación. El lenguaje "parece quedarse corto" frente a la complejidad o "la realidad percibida" del fenómeno, no existe, para ellos, el significante que cierre el signo lingüístico en su totalidad.

Si continuamos considerando que la hipótesis es una nueva representación de las evidencias disponibles, si además asumimos que esas representaciones (en las formas que adopte) se convierten (o traducen) en un enunciado verbal, hay un trabajo intelectual complejo que no debemos subestimar. Desde nuestra perspectiva, es la conversión de esas imágenes o representaciones mentales de la evidencia, en palabras o códigos lingüísticos, que bien sabemos son arbitrarias y pueden tener poco o nada que ver con la cosa designada. Si es cierto que esto ocurre, el obstáculo también parece ser de naturaleza ontogenética.

\footnotetext{
${ }^{19}$ Esta última frase proviene de una anécdota del autor Vosgerau (2012), a quien un interlocutor le comentó que lo que veía escrito en su papel era una oración y no un pensamiento. Aunque la oración lo refleje, no es el pensamiento en sí.
} 
La naturaleza ontogenética, psicológica y epistemológica de los errores y obstáculos en la construcción de hipótesis científicas: un estudio de caso

\section{Conclusiones}

Entender las dificultades de los estudiantes para construir una hipótesis científica nos permite también crear las estrategias didácticas adecuadas para mitigar sus efectos y dar paso al añorado pensamiento lógico-argumentativo.

Las razones a las que hemos apelado parecen bastante plausibles para explicar estas dificultades. Son varias y de diversa naturaleza. Por ello, nos corresponde, ante cada caso, identificar cuál de estas razones es la que predomina y cuáles podemos considerar "universales" para crear estrategias que permitan superar tales obstáculos. Así como la neuropsicología ha creado estrategias para inhibir la supremacía del sesgo de emparejamiento perceptivo y con ello facilitar el paso al pensamiento lógico ${ }^{20}$, así es necesario crear otras que mitiguen la supremacía de lo intuitivo, de las emociones "en cantidades inadecuadas", que promuevan la independencia psicológica de la autoridad, que disminuyan el efecto de las arbitrariedades del lenguaje y la acrítica estabilidad de nuestra identidad.

Un diseño didáctico-psicológico, que lleve a cabo las acciones antes recomendadas, será mucho mejor que eliminar las disciplinas formadoras del pensamiento lógico-argumentativo de los programas de estudio. Bien sabemos que quienes consolidan este pensamiento logran generar hipótesis sin temor alguno, generalmente bien construidas, promueven el diálogo y permiten el acceso a nuevos conocimientos. Una vez superados los obstáculos para construir una hipótesis, corresponde probar lo que se afirma.

\section{Agradecimientos}

Agradecemos el valioso apoyo del proyecto Epistemologías personales en docentes y su impacto para la enseñanza de la ciencia y de la filosofía (UNAM, DGAPA-PAPIIT IN 400319).

\footnotetext{
${ }^{20}$ Ante la tarea de identificar en un conjunto de figuras geométricas con diversos colores las combinaciones que señalaran que la regla "Si no hay un cuadrado rojo a la izquierda entonces hay un círculo amarillo a la derecha" era falsa, y en la que más de 90\% de los sujetos emitieron una respuesta incorrecta, Houde y Cols (2003) concluyen que entran en conflicto las estrategias perceptivas con las lógicas. Los sujetos centran su atención en las formas y colores más que en la consigna. Houde y Cols (2003) deciden inhibir las estrategias perceptivas impulsivas poniendo al sujeto sobre aviso de las trampas del razonamiento tendidas por la percepción. Este sobre aviso produjo un revés en los resultados, en esta ocasión más del $90 \%$ de los sujetos acertó en su respuesta.
} 


\section{$\underline{\text { Referencias }}$}

Bachelard, G. (2003). La formación del espíritu científico. Buenos Aires: Siglo XXI.

Barceló, A. (2005). Lógica y Lenguaje. Disponible en http://www.filosoficas.unam.mx/ abarcelo/PDF/Logica_y_Lenguaje.pdf

Barceló, A. (2012). Words and images in argumentation, Argumentation, 26 (3), pp. 355-368.

Beuchot, M. (2008). Introducción a la lógica. México, Universidad Nacional Autónoma de México.

Brousseau, G. (2003). Erreurs, difficultés, obstacles, texte propose comme annexe pour le cours : Situations fundamentales et processus génétiques de la statisque. Présenté lors de la XII école d'été de didactique des mathématiques.

Brun, G, Doğuoğlu, U, y Kuenzle, D. (2008): Introduction: A new role for emotions in epistemology? En Brun, G., Doğuoğlu, U., Kuenzle, D. (Eds). Epistemology and Emotions. Aldershot, Ashgate Publishing, Ltd., 1-32.

Copi, I. (1979). Lógica simbólica. México: CECSA.

Couló, A. (2017). Los obstáculos epistemológicos en la enseñanza de la lógica. En Z. Monroy, R. León y G. Alvarez (Eds.) Obstáculos epistemológicos en la enseñanza y el aprendizaje de la filosofía y de la ciencia. México: Facultad de Psicología, UNAM, 117-130.

Charnay, R, y Mante, M. (1992). De l'analyse d'erreur en mathématiques aux dispositifs de remédiation, Repères, (7), 5-32.

Damasio, A. (2007). El error de Descartes. La emoción, la razón y el cerebro humano. Barcelona: Crítica.

De Sousa, R. (2008). Epistemic feelings. En Brun, G, Doğuoğlu, U, Kuenzle, D. (Eds.). Epistemology and emotions. Aldershot, Ashgate Publishing, Ltd, 185-205.

Echevarría, J. (1998). Similaridades, isomorfismos y homeomorfismos entre representaciones científicas (Similitudes, Isomorfisms and Homeomorfisms Among Scientific Representations). Theoria. Revista de Teoría, Historia y Fundamentos de la Ciencia, 13(1), 89-112.

Elgin, C. (2008). Emotion and Understanding. En Brun, G, Doğuoğlu, U, Kuenzle, D. (Eds). Epistemology and Emotions, Aldershot, Ashgate Publishing, Ltd, 33-50.

Eraña, A. (2003). Normatividad epistémica y estructura heurística del razonamiento. Crítica: Revista Hispanoamericana de Filosofía, 35(104), 69-108. 
Evans, J. (1989). Bias in human reasoning: Causes and consequences. Lawrence Erlbaum Associates, Inc.

Garrido, I. (2013). Crítica de libros: Pensar rápido. Pensar despacio. Esic Market Economics and Business Journal, 44(3), 205-209. Recuperado en https://www.esic.edu/documentos/revistas/esicmk/130912_131902_E.pdf

Giere, R. (1992). La explicación de la ciencia: Un acercamiento cognoscitivo. México: Consejo Nacional de Ciencia y Tecnología. Original en inglés de 1988.

Guillaumin, G. (2009). El relativismo epistemológico visto a través de la teoría del cambio científico de Thomas Kuhn. Relaciones. Estudios de historia y sociedad, 30(120), 139-164.

Hacking, I. (1993). Representing and Intervening. Cambridge, Cambridge Univ. Press

Hempel, C. G. (1988). La explicación científica: estudios sobre la filosofía de la ciencia. Barcelona, España, Paidós.

Hempel, C. G. (1973/2003). Filosofía de la ciencia natural. Madrid, Alianza Universidad.

Hookway, C. (2008). "Epistemic immediacy, doubt and anxiety: On a role for affective states in epistemic evaluation", en Brun, G, Doğuoğlu, U, Kuenzle, D. (eds). Epistemology and Emotions, Aldershot, Ashgate Publishing Ltd., pp. 51-66.

Houde, O., Moutier, S., Zago, L., y Tzourio-Mazoyer, N. (2003). "Corrección de los errores de razonamiento", Mente y Cerebro, Enero-Marzo (2), pp. 62-70.

Ibarra, A. Y Mormann, T. (1997). “Theories as representations”, Poznan studies in the philosophy of the sciences and the humanities, 61, pp. 59-97.

Kahneman, D. (2012). Pensar rápido, pensar despacio. España, Debate.

Kuhn, D, Cheney, R, y Weinstock, M. (2000). "The development of epistemological understanding", Cognitive development, 15(3), pp. 309-328.

Linch, M. Y Woolgar, S (eds). (1990). Representations in scientific practice. Cambridge Mass, MIT Press

Lorenzano, P., y Ginnobili, S. (2015): "Reconstrucción de patrones explicativos vs. Reconstrucción de teorías", en Filosofía e Historia de la Ciencia en el Cono Sur. Selección de trabajos del IX Encuentro y las XXV Jornadas de Epistemología e Historia de la Ciencia, Córdoba, Editorial de la Universidad Nacional de Córdoba, pp. 417- 428.

Lorenzano, P. (2004): Filosofía de la Ciencia, Bernal: Universidad Nacional de Quilmes, 135 pp., 2004. 
Monroy, Z. (2017): "El sentido común como obstáculo para la enseñanza y el aprendizaje de la lógica”, en Z. Monroy, R. León y G. Álvarez, Obstáculos epistemológicos en la enseñanza y el aprendizaje de la filosofía y de la ciencia: México, Facultad de Psicología, UNAM, 2017, pp.105 -116.

Nagel, E. (1981): La estructura de la ciencia. Barcelona, Buenos Aires, México, Paidós.

Olshanski, S., Carnes, A., Butler, N. (2001): “Anatomía de larga vida”, Investigación y Ciencia, Mayo (296), pp. 44-49.

Palau, G, y Couló, A. (2011). Systematic errors as an input for teaching logic. En Tools for Teaching Logic, TICTTL 2011. Lecture Notes in Computer Science, v6680. Springer, Berlin, Heidelberg, 183-189.

Palau, G. (2014). Lógica formal y argumentación como disciplinas complementarias. MISC. Disponible en:

http://www.memoria.fahce.unlp.edu.ar/libros/pm.359/pm.359.pdf

Perelman, CH., \& Olbrecht Tyteca, L. (2006). Tratado de la Argumentación. La nueva Retórica. España: Editorial Gredos.

Pérez Ransanz, A. R. (2005). Las emociones como ingrediente básico de nuestras representaciones. Representaciones. Revista de Estudios sobre Representaciones en Arte, Ciencia y Filosofía, SIRCA Publicaciones Académicas, (1), 121- 134.

Posner G., Strike, K., Hewson, P. Y Gertzog, W. (1982). Accommodation of a Scientific Conception: Toward a Theory of Conceptual Change. Science Education, 66(2), 211- 227

Reñón, L. (2003). Si de argumentar se trata. España. Editorial Montesinos.

Saussure, F. (1945). Curso de lingüística general. Buenos Aires: Editorial Losada.

Stincer, D, y Blum, B. (2017). El modelo argumentativo de Toulmin y la eficacia de titulación. Revista Electrónica de Investigación Educativa, 19(4), 9-19.

Thagard, P. (2002). The Passionate Scientific: Emotion in Scientific Cognition. En P. Carruthers, S. Stich \& M. Siegal (Eds.). The cognitive basis of science, Cambridge, Cambridge University Press, pp. 235-250.

Thagard, P. (2008): "How Cognitions Meets Emotion: Beliefs, Desires, and Feelings as Neural Activity", en Brun, G, Doğuoğlu, U, Kuenzle, D. (eds). Epistemology and Emotions, Aldershot, Ashgate, Publishing, Ltd. 167-184.

Toulmin, S. (2003). The uses of argument. Cambridge. University Press. 
La naturaleza ontogenética, psicológica y epistemológica de los errores y obstáculos en la construcción de hipótesis científicas: un estudio de caso

Van Eemeren F. H. \& Grootendorst, R. (2004). A systematic theory of argumentation. The pragmadialectical approach. Cambridge University Press.

Van Fraassen, B. C. (1980). The scientific image. Oxford, Blackwell, trad. Castellana en La imagen científica, México, UNAM, 1996.

Van Dijk, T. (1992). La ciencia del texto. Barcelona, España: Ediciones Paidós.

Van Dijk, T. (1998). Texto y contexto: semántica y pragmática del discurso. Madrid: Cátedra.

Vosgerau, G. (2012). El lenguaje y la razón. Investigación y Ciencia, Octubre(433), 84-87.

Wason, P. C. (1960). On the failure to eliminate hypotheses in a conceptual task. Quarterly Journal of Experimental Psychology, 12, 129-140. 